\title{
Why do children perseverate when they seem to know better: Graded working memory, or directed inhibition?
}

\author{
Nicholas J. Cepeda \\ University of California, San Diego, California \\ and University of Colorado, Boulder, Colorado \\ AND \\ Yuko Munakata \\ University of Colorado, Boulder, Colorado
}

\begin{abstract}
Children sometimes have trouble switching from one task to another, despite demonstrating an awareness of current task demands. This behavior could reflect problems either directly inhibiting previously relevant information or sufficiently activating graded working memory representations for the current task. We tested competing predictions from each account, using a computerized card-sorting task in which we assessed children's task switching abilities and their response speed to simple questions about current task demands. All children answered these questions correctly, but children who successfully switched tasks responded more quickly to questions than did children who perseverated on previous tasks, even after factoring out processing speed and age. This reaction time difference supports graded working memory accounts, with stronger representations of current task demands aiding both task-switching and responses to questions. This result poses a challenge for directed inhibition accounts, because nothing needs to be inhibited to answer simple questions that lack conflicting information.
\end{abstract}

Individuals often perseverate: on casual clothes Friday, they put on formal clothes; switching between computer operating systems, they press the wrong control key. Perseveration is a hallmark of ADHD (Barkley, 1997), autism (Tsuchiya, Oki, Yahara, \& Fujieda, 2005), and frontal lobe damage (Milner, 1963). It is particularly salient in typically developing preschoolers and young children: When asked to change from one matching rule (e.g., shape) to a new one (e.g., color), young children often continue to use the first rule (Kirkham, Cruess, \& Diamond, 2003; Perner \& Lang, 2002; Towse, Redbond, Houston-Price, \& Cook, 2000; Zelazo, Müller, Frye, \& Marcovitch, 2003), even after feedback is provided (Cepeda, Cepeda, \& Kramer, 2000; Yerys \& Munakata, 2007) and despite clear and repeated instruction. In sharp contrast, young children are very good at answering questions about what they are supposed to be doing (e.g., "In the shape game, where do cats go?"-Zelazo \& Reznick, 1991).

Why do children perseverate, when they seem to know better? We contrast two explanations, and test their distinct predictions in the current study. According to directed inhibition accounts (Anderson, 2005; Freud, 1938/1995; James, 1901; Ridderinkhof, van den Wildenberg, Wijnen, \& Burle, 2004; Zacks \& Hasher, 1994), people perseverate due to problems with top-down inhibition of prepotent responses (e.g., old stimulus-response rules). They answer questions correctly because they know what action they should perform, yet they cannot prevent themselves from using the old rule. Many behaviors that seem to require directed inhibition may be explicable in other ways (Altmann, 2004; Deák, 2003; Egner \& Hirsch, 2005; Hedden \& Park, 2003; MacLeod, Dodd, Sheard, Wilson, \& Bibi, 2003; Stedron, Sahni, \& Munakata, 2005). For example, according to graded working memory accounts (Cohen, Dunbar, \& McClelland, 1990; Roberts \& Pennington, 1996), people perseverate due to problems strongly maintaining current task information (e.g., the new rule) in working memory. They answer questions correctly because weak working memory representations of current task rules suffice for "nonconflict" tasks where information about the old rule is removed (Morton \& Munakata, 2002). Consistent with this, apparent knowledge-action dissociations disappear when measures are equated for degree of conflict (e.g., sorting a red cat and answering the question "Where do red cats go?"; Munakata \& Yerys, 2001). Under graded memory accounts, inhibition emerges indirectly from competition throughout the system, and representations with more working memory support are more likely to win.

Graded working memory and inhibition accounts are difficult to distinguish under conflict conditions (MacLeod et al., 2003; Mayr, 2002), because results could be

N. J. Cepeda, ncepeda@yorku.ca 
attributed to either actively inhibiting an old task or actively maintaining a new task. A critical test is what happens under nonconflict conditions (e.g., answering questions like "In the shape game, where do cats go?"). Both directed inhibition and graded working memory accounts can explain how children can answer such questions correctly, regardless of whether they sort cards correctly. Either knowing exactly what to do (as per the directed inhibition account) or having weak memory representations of the current rule (as per the graded working memory account) would produce correct responses to these nonconflict questions. However, only the graded memory account predicts that children who are able to switch tasks should show faster responses to nonconflict questions. Children who are able to switch tasks should have stronger working memory representations of the current rule, compared to those who perseverate, which should support faster performance when answering nonconflict questions about that rule. In contrast, under directed inhibition, the ability to switch should not correlate with response time to nonconflict questions, because there is nothing to inhibit.

In this study, we compared how quickly switchers and perseverators answered nonconflict questions about the current rule. To assess whether differences observed in nonconflict reaction times were due to general processing speed differences (thought to reflect general neural speed and efficiency) between switchers and perseverators (Cepeda, Kramer, \& Gonzalez de Sather, 2001; Salthouse, Fristoe, McGuthry, \& Hambrick, 1998), we included measures that allowed processing speed to be factored out.

\section{METHOD}

\section{Participants}

A total of 32 participants began the study. Six participants were excluded from analysis: Two perseverated during the second block and switched during the third block, 1 (incorrectly) matched by color during the first block, 1 matched indiscriminately during the first and subsequent blocks, 1 failed to meet the preswitch accuracy criterion (because this participant failed to tap the screen as instructed), and 1 chose to end the study before completion. Participants included in the analyses ranged in age from 5.74 to 6.86 years old. We focused on this age because it produced $50 \%$ perseverators using this version of the card sorting paradigm. Mean age of participants who switched tasks was 6.45 years old ( $S D=0.36, n=13,5$ female), and that of those who perseverated was 6.29 years old $(S D=0.34, n=13,7$ female). Between groups, age did not differ $[t(24)=1.2, p=.243]$. The finding that half the children switched and half perseverated, at a fixed age, is typical of children's card sorting studies (Bialystok \& Martin, 2004; Deák, 2003; Kirkham et al., 2003; Munakata \& Yerys, 2001; Noble, Norman, \& Farah, 2005; Perner \& Lang, 2002; Towse et al., 2000; Zelazo et al., 2003).

\section{Procedure and Design}

Computerized card sorting. The card sorting paradigm (Deák, 2003) required participants to switch between three different tasks: matching by shape (Block 1), color (Block 2), or size (Block 3). Other studies (Deák, Narasimham, Cepeda, \& Legare, 2007) have established that children perseverate on the first task dimension regardless of whether it is shape, color, or size. In each of three blocks, participants were (1) asked to identify targets using the about-to-be-introduced task dimension, (2) introduced to the current task, (3) presented nonconflict stimuli, and (4) presented conflict stimuli. The purpose of these four segments was to (1) assess general speed of making responses, (2) ac- tivate working memory representations, (3) assess working memory strength for the task rule, and (4) assess ability to match correctly. Conflict trials were presented after nonconflict trials so that we could obtain a purer measure of working memory strength; if we had switched the order, perseverators would have had to switch tasks sets in order to answer the nonconflict questions, thereby producing slowed nonconflict trial RTs for reasons other than working memory strength.

The top half of the screen consisted of a large blue cat, small yellow fish, and medium red bird, each of which was surrounded by a black box (Figure 1). A horizontal bar separated these three "target" boxes from a "stimulus" box. Target boxes turned green when selected. Almost all instructions were presented as prerecorded videos (lower left of screen, in place of the filled gray box shown in Figure 1) that showed the talking head of a female undergraduate student. Responses were made using a MagicTouch touch screen.

For an overview of the study design and trial examples, see the Appendix. Before the three card sorting blocks began, participants were familiarized to the touch screen. After touch screen practice, three "sorting" blocks (shape, color, and size) were presented.

Each block began by asking participants to identify each target stimulus (e.g., "Can you press the small one [fish, yellow one]?"). Reaction time was computed as time from end of instruction video clip to first touch-screen response. Individual participants tended to respond using the same hand, starting from the same location, throughout the study, although starting position differed across participants. These identity trials provided a way to factor out general neural speed, perceptual processing speed, motor speed, and individual differences in response style.

Immediately following target identification, the task was introduced (e.g., "Now we're going to play the size game. In the size game, when you see a small one, press the small one. Can you press the small one?"), and participants again identified each relevant stimulus dimension. These trials were designed to activate the current task in working memory.

Immediately following task introduction, nonconflict questions were asked about the task (e.g., "In the size game, show me what you press when you see a small one.") (Figure 1). ${ }^{1}$ We believe that these trials provide a measure of working memory strength, or the degree to which the current rule dominates working memory. The more strongly the rule is held in working memory, the more quickly participants should respond on these trials.

Across these three phases (target identification, task introduction, and nonconflict trials), at the start of each block, children thus received ample opportunity to detect the rule and to demonstrate that they had done so. Specifically, across these phases, children heard the rule 7 times and each of the three features relevant to that rule 5 times, and they could use the rule 12 times.

Immediately following nonconflict trials, conflict trials were presented. Each conflict trial began with the question, "Okay! Which do you press for this one?," followed by stimulus presentation. ${ }^{2}$ These trials assessed whether participants could match the stimulus to a target using the current rule. Each stimulus matched each target card on only one dimension - shape, color, or size — making the stimuli response incompatible across three dimensions. All six possible stimulus cards that meet these criteria (e.g., small red cat) were used. In addition to "standard" conflict trials (Figure 1), "novel" conflict trials, which used a medium purple octopus, large orange rabbit, and small green owl as targets, and corresponding new single-match-dimension stimuli, were presented. A set of three "identical" stimuli, which looked identical to target stimuli, was also presented during the conflict trial portion of each block. ${ }^{3}$ During each conflict trial segment, first six standard trials were presented, followed by three identical trials, followed by six standard trials, followed by six novel trials. Preceding novel trials, participants were told, "Good job! Just keep doing what you were doing." Stimuli were presented in a pseudorandom order (constant across participants), which differed during each subblock, so that the order of stimulus presentation was (to the participant) unpredictable.

Processing speed. In this study, nonconflict trial RTs are treated as a potential measure of working memory strength. Factoring out 


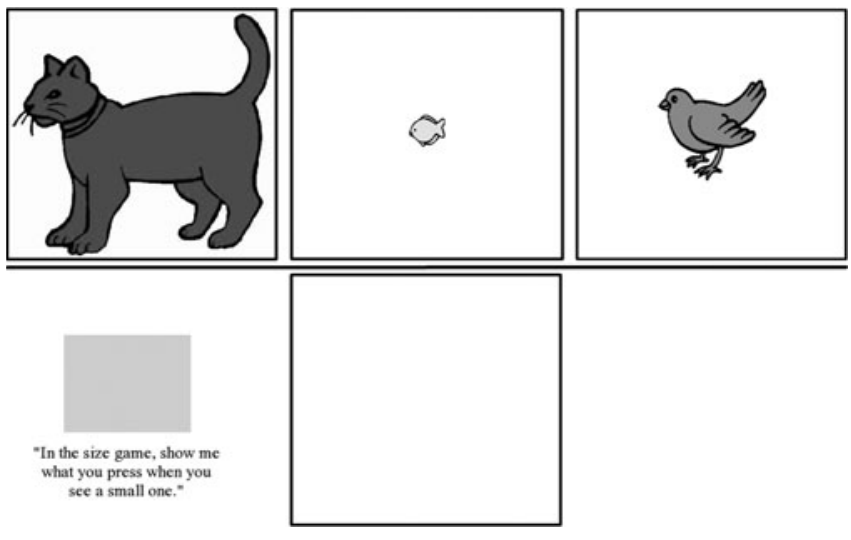

Figure 1. An example nonconflict question trial in the computerized card-sorting task. Text was spoken during video clip presentation, prior to stimulus display or touch-screen response. Dark, medium, and light gray correspond to blue, red, and yellow, respectively.

processing speed allows the removal of extraneous influences on this measure, including general neural speed, perceptual processing speed, motor speed, and individual differences in response style. Target identification trials (described above) and the box completion task provided two measures of processing speed. The box completion task (Salthouse, 1996) consisted of a $5 \times 7$ array of three-sided, 2-D boxes (e.g., that contained a left, top, and right side), $1.9 \mathrm{~cm}$ on each side, printed on a letter-sized sheet of paper. The fourth side of each box (e.g., the bottom side) was drawn as quickly as possible, using a crayon. The number of boxes completed in $30 \mathrm{sec}$ was measured.

\section{Data Trimming}

Computerized card sorting. Only correct-response trials were used in reaction time analyses. RTs shorter than $200 \mathrm{msec}$ were eliminated (without replacement). This resulted in the loss of $2.1 \%$ of trials. For trimming of extremely long or short RTs, we followed a modified version of the trimming method used by Friedman and Miyake (2004). ${ }^{4}$ Following the trimming procedure, we computed mean RTs for each participant for each condition, and these mean RTs were used in analyses.

Processing speed. No box completion scores lay more than $3 S D$ from the mean of remaining scores, so these data were not trimmed.

\section{RESULTS}

All participants successfully matched at least 15 of 18 preswitch conflict stimuli and then fell into discrete "switcher" and "perseverator" categories on subsequent blocks. Out of 36 postswitch conflict stimuli, perseverators correctly matched 0 to $4(M=1.6, S D=1.3)$, and switchers correctly matched 25 to $36(M=32.2, S D=3.3)$. Errors were largely perseverative first-task responses $(97.8 \%$ of perseverators' errors; $83.5 \%$ of switchers' errors).

Switchers and perseverators did not differ in processing speed, on either the box completion task [number of boxes completed: perseverators, $M=23.7, S D=4.5$; switchers, $M=26.1, S D=4.1 ; t(24)=1.4, d=.56, p=.170]$, or in target identification RT performance [perseverators, $M=881 \mathrm{msec}, S D=237,100 \%$ correct; switchers, $M=$ $743 \mathrm{msec}, S D=221,98.3 \%$ correct; $t(24)=1.5, d=$ $.60, p=.140]$. However, both processing speed measures showed nonsignificant advantages for switchers. We thus include processing speed (along with age) as a covariate in our working memory strength analyses, to remove individual differences in these factors and focus on the relationship of interest between switch status and nonconflict RT. (Controlling for age, the partial correlation between working memory strength and processing speed was $r=$ .11 , suggesting that our processing speed and working memory strength measures tap independent constructs.) We also assess the relative contributions of processing speed and working memory strength on switching.

On nonconflict questions, switchers and perseverators were equally highly accurate $(p=.13)$ (Figure 2). Qualitatively, switchers were faster on nonconflict questions, within every block. We examined nonconflict question data quantitatively, using a mixed-model ANCOVA that included block (shape, color, or size) as a within-subjects factor, switch status (switcher or perseverator) as a between-subjects factor, and age and processing speed (average $z$ score for box completion score and target identification RT) as covariates. ${ }^{5}$ There was a significant main effect of switch status $[F(1,20)=5.3, d=0.97, p<.05]$. All other main effects and interactions were nonsignificant (all $p \mathrm{~s}>.27$ ). Thus, switchers were faster than perseverators on nonconflict questions, even after factoring out processing speed and age, as predicted by graded working memory but not directed inhibition accounts.

To further assess contributions of age, working memory strength, and general processing speed, we performed a hierarchical logistic regression, with switch status as a dependent variable, first entering age, then processing speed, and then working memory strength (average nonconflict question RT) (Table 1). Consistent with the prior ANCOVA analysis, results suggest that working memory strength is linked to ability to switch tasks. In fact, working memory strength is nearly four times better than processing speed at predicting switch status.

\section{DISCUSSION}

Switchers were faster than perseverators at answering nonconflict questions, even after factoring out processing speed and age. This effect was present to the same degree across shape, color, and size dimensions, indicating that it was quite general. These results challenge the idea that switchers and perseverators both know exactly what to do, but perseverators simply cannot prevent themselves from carrying out previous behaviors. Instead, these results are consistent with the idea that switchers have stronger working memory representations of the current task than do perseverators, which support both successful switching and faster responses to nonconflict questions. To our knowledge, this study provides the first behavioral test of competing predictions from graded working memory and directed inhibition accounts of perseveration.

Could a directed inhibition account be revised to explain these findings? Perhaps reaction times to nonconflict questions benefit from certain kinds of inhibition, even though conflicting information has been removed. For example, children may have general activation of prior task-relevant information (e.g., the color red). Alterna- 

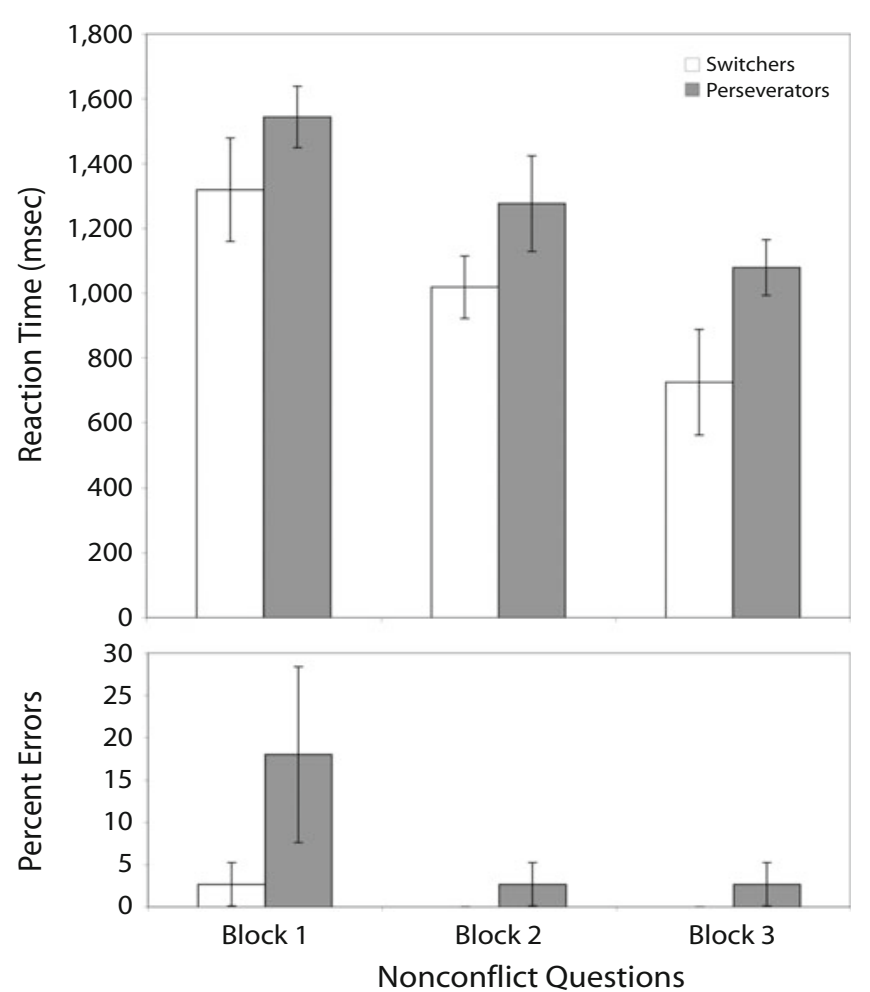

Figure 2. Nonconflict question trial accuracies and correctresponse RTs (1 SEM), by block. Although both switchers and perseverators can answer nonconflict questions accurately, switchers answer reliably faster than perseverators, even after factoring out processing speed and age.

tively, the targets may be viewed as providing conflicting information, even though conflict has been removed from the questions themselves. In each case, the argument is that better abilities at inhibiting such conflicting information leads to faster responses. However, these revised inhibitory accounts predict that the reaction time difference between switchers and perseverators should be smallest on the first block, before conflicting associations or activations build. Our data show no hint of an interaction between block and switch status $(F<1$, Figure 2). Thus, our data do not support these revised inhibitory accounts. In contrast, these data are consistent with graded working memory accounts: Stronger working memory representations of the current task can support faster performance across different levels of conflicting information, thus aiding performance similarly across blocks.

Table 1

Hierarchical Logistic Regression Predicting Switch Status (Switcher or Perseverator)

\begin{tabular}{llllc}
\hline \multicolumn{1}{c}{ Independent Variable } & Wald & $p$ & $R^{2}$ & $\begin{array}{c}R^{2} \\
\text { Change }\end{array}$ \\
\hline Age & 0.35 & .56 & .074 & \\
Processing speed & 2.28 & .13 & & .067 \\
Working memory strength & 3.30 & .07 & & .251 \\
\hline
\end{tabular}

Note-Nagelkerke pseudo- $R^{2}$ values are provided.
Of course, evidence for graded working memory does not rule out the possibility that a top-down, directed inhibitory control mechanism exists, and may contribute to performance on some executive function tasks. One could argue, for example, that directed inhibition serves as an additional process to the graded working memory processes for which we provide evidence here. However, in many cases, the evidence used to argue for an additional directed inhibition process can be explained and modeled more parsimoniously in terms of graded working memory, with inhibition emerging indirectly from competition throughout the system (Cohen et al., 1990; Morton \& Munakata, 2002; Stedron et al., 2005). For example, the fact that people can perseverate despite answering nonconflict questions correctly has been viewed as requiring an inhibitory explanation. However, the current findings confirm a distinguishing prediction from graded working memory accounts of this phenomenon.

Similarly, evidence for graded working memory does not rule out other accounts of perseveration. For example, cognitive complexity and control (CCC) theory posits that switchers and perseverators differ in their ability to consciously reflect on tasks and recognize higher order rules (Zelazo et al., 2003). This account may not be able to explain the current findings of differences in nonconflict question RT, because only conflicting tasks are posited to require conscious reflection and recognition of higher order rules. However, one could argue that graded working memory explains the current findings, but CCC factors are at work in addition to graded working memory. It is not clear though whether these factors add explanatory power (Yerys \& Munakata, 2006).

One might ask whether the current results could alternatively be explained more directly in terms of the consequences of response times. Specifically, perseverators' slower response times (as assessed on nonconflict trials) might allow more conflict to build on conflict trials, leading to more errors. Switchers can respond more quickly, before this conflict builds. Although we found no differences between perseverators and switchers in their reaction times on our processing speed measures, both measures showed nonsignificant advantages for switchers. We believe this account is unlikely, for two reasons. First, the working memory strength and processing speed measures seem to tap different constructs, and working memory strength is nearly four times better than processing speed at predicting switch status. Second, this account assumes that slower responding allows more conflict to build. We do not know of any evidence that conflict builds in this way, and evidence from other paradigms suggests otherwise (e.g., Glaser \& Glaser, 1982).

Remarkably, a 308-msec difference in nonconflict question RT for switchers versus perseverators was predictive of whether children switched to a new task, even after factoring out processing speed and age. This finding suggests that graded working memory influences task switching ability, where children with stronger working memory representations of a task receive an RT benefit on nonconflict trials and an accuracy benefit on conflict trials. Whether 
this conclusion holds true for other tasks and populations remains to be tested. We believe that the nonconflict RT measure introduced here provides an important new tool that should allow researchers to assess contributions from working memory strength across a range of executive control tasks and across individuals of various ages.

\section{AUTHOR NOTE}

This work was supported by Grant R01 HD37163 from NICHD. We thank Sara McQuiston for providing the voice and face of the card sorting task, Chris Chatham, Adele Diamond, and Akira Miyake for useful discussions, and members of the Cognitive Development Center for assisting with this study. Correspondence concerning this article should be addressed to N. J. Cepeda, York University, Department of Psychology, 4700 Keele Street, Toronto, ON, M3J 1P3 Canada (e-mail: ncepeda@yorku.ca).

\section{REFERENCES}

Altmann, E. M. (2004). Advance preparation in task switching: What work is being done? Psychological Science, 15, 616-622.

Anderson, M. C. (2005). The role of inhibitory control in forgetting unwanted memories: A consideration of three methods. In N. Ohta, C. M. MacLeod, \& B. Uttl (Eds.), Dynamic cognitive processes (pp. 159-190). New York: Springer.

BARKLEY, R. A. (1997). Behavioral inhibition, sustained attention, and executive functions: Constructing a unifying theory of ADHD. Psychological Bulletin, 121, 65-94.

Bialystok, E., \& Martin, M. M. (2004). Attention and inhibition in bilingual children: Evidence from the dimensional change card sort task. Developmental Science, 7, 325-339.

Cepeda, N. J., Cepeda, M. L., \& Kramer, A. F. (2000). Task switching and attention deficit hyperactivity disorder. Journal of Abnormal Child Psychology, 28, 213-226.

Cepeda, N. J., Kramer, A. F., \& Gonzalez de Sather, J. C. M. (2001). Changes in executive control across the life-span: Examination of task switching performance. Developmental Psychology, 37, 715-730.

Cohen, J. D., Dunbar, K., \& McClelland, J. L. (1990). On the control of automatic processes: A parallel distributed processing model of the Stroop effect. Psychological Review, 97, 332-361.

DEÁK, G. O. (2003). The development of cognitive flexibility and language abilities. In R. Kail (Ed.), Advances in child development and behavior (Vol. 31, pp. 271-327). San Diego: Academic Press.

Deák, G. O., Narasimham, G., Cepeda, N. J., \& Legare, C. (2007). Cognitive flexibility in young children: Age, individual, and task differences. Manuscript in preparation.

Egner, T., \& Hirsch, J. (2005). Cognitive control mechanisms resolve conflict through cortical amplification of task-relevant information. Nature Neuroscience, $\mathbf{8}, 1784-1790$.

FREUD, S. (1995). The basic writings of Sigmund Freud (A. A. Brill, Trans., \& Ed.). New York: Random House. (Original work published 1938)

Friedman, N. P., \& MiYaKe, A. (2004). The relations among inhibition and interference control functions: A latent-variable analysis. Journal of Experimental Psychology: General, 133, 101-135.

Glaser, M. O., \& Glaser, W. R. (1982). Time course analysis of the Stroop phenomenon. Journal of Experimental Psychology: Human Perception \& Performance, 8, 875-894.

HEDDEN, T., \& PARK, D. C. (2003). Contributions of source and inhibitory mechanisms to age-related retroactive interference in verbal working memory. Journal of Experimental Psychology: General, 132, 93-112.

JAMES, W. (1901). Talks to teachers on psychology and to students on some of life's ideals. Whitefish, MT: Kessinger.

Kirkham, N. Z., Cruess, L., \& Diamond, A. (2003). Helping children apply their knowledge to their behavior on a dimension-switching task. Developmental Science, 6, 449-476.

MacLeod, C. M., Dodd, M. D., Sheard, E. D., Wilson, D. E., \& BIBI, U. (2003). In opposition to inhibition. In B. H. Ross (Ed.), The psychology of learning and motivation (Vol. 43, pp. 163-214). San Diego: Academic Press.

MAYR, U. (2002). Inhibition of action rules. Psychonomic Bulletin \& Review, 9, 93-99.
Milner, B. (1963). Effects of different brain lesions on card sorting. Archives of Neurology, 9, 90-100.

Morton, J. B., \& Munakata, Y. (2002). Active versus latent representations: A neural network model of perseveration, dissociation, and decalage. Developmental Psychobiology, 40, 255-265.

Munakata, Y., \& Yerys, B. E. (2001). When dissociations between knowledge and action disappear. Psychological Science, 12, 335-337.

Noble, K. G., Norman, M. F., \& Farah, M. J. (2005). Neurocognitive correlates of socioeconomic status in kindergarten children. Developmental Science, 8, 74-87.

Perner, J., \& LANG, B. (2002). What causes 3-year-olds' difficulty on the dimensional change card sorting task? Infant \& Child Development, 11, 93-105.

Ridderinkhof, K. R., van den Wildenberg, W. P. M., WiJnen, J., \& BurLe, B. (2004). Response inhibition in conflict tasks is revealed in delta plots. In M. I. Posner (Ed.), Cognitive neuroscience of attention (pp. 369-380). New York: Guilford.

Roberts, R. J., JR., \& Pennington, B. F. (1996). An interactive framework for examining prefrontal cognitive processes. Developmental Neuropsychology, 12, 105-126.

SAlthouse, T. A. (1996). The processing speed theory of adult age differences in cognition. Psychological Review, 103, 403-428.

Salthouse, T. A., Fristoe, N., McGuthry, K. E., \& Hambrick, D. Z. (1998). Relation of task switching to speed, age, and fluid intelligence. Psychology \& Aging, 13, 445-461.

Stedron, J. M., Sahni, S. D., \& Munakata, Y. (2005). Common mechanisms for working memory and attention: The case of perseveration with visible solutions. Journal of Cognitive Neuroscience, 17, 623-631.

Towse, J. N., Redbond, J., Houston-Price, C. M. T., \& Cook, S. (2000). Understanding the dimensional change card sort: Perspectives from task success and failure. Cognitive Development, 15, 347-365.

Tsuchiya, E., OKI, J., Yahara, N., \& Fujieda, K. (2005). Computerized version of the Wisconsin card sorting test in children with highfunctioning autistic disorder or attention-deficit/hyperactivity disorder. Brain \& Development, 27, 233-236.

Yerys, B. E., \& Munakata, Y. (2006). When labels hurt but novelty helps: Children's perseveration and flexibility in a card-sorting task. Child Development, 77, 1589-1607.

Yerys, B. E., \& Munakata, Y. (2007). The effects of negative feedback on children's flexibility. Manuscript in preparation.

ZACKS, R. T., \& HASHER, L. (1994). Directed ignoring: Inhibitory regulation of working memory. In D. Dagenbach \& T. H. Carr (Eds.), Inhibitory mechanisms in attention, memory, and language (pp. 241264). New York: Academic Press.

Zelazo, P. D., Müller, U., Frye, D., \& Marcovitch, S. (2003). The development of executive function. Monographs of the Society for Research in Child Development, 68, 11-27.

Zelazo, P. D., \& Reznick, J. S. (1991). Age-related asynchrony of knowledge and action. Child Development, 62, 719-735.

\section{NOTES}

1. These nonconflict questions were followed by three visual nonconflict trials that were designed to be nonconflicting, but were dropped from the analyses because of possible conflicts. For example, for the shape nonconflict stimuli, each shape was presented with a white fill color and a medium-large size. Because the medium-large stimuli could have been perceived as medium or large, they might be viewed as providing conflicting size information. For this reason, we focus our analyses on the nonconflict questions, where this issue does not apply.

2. The word "okay" is commonly used as neutral feedback, to keep children interested in the task and to prevent them from getting discouraged. The wording was used consistently across switchers and perseverators, so it cannot explain differences between these groups.

3. Identical and novel trials were included for reasons that are not central to the current paper. Identical trials are not discussed further. Novel and standard conflict trial accuracies did not differ, so these trials were combined for measuring switching ability.

4. First, all RTs faster than $200 \mathrm{msec}$ were removed. Second, all RTs more than $3 S D$ s shorter or longer than the mean of remaining RTs were removed. Third, these empty cells were replaced with a value that was 
$3 S D$ s shorter or longer than the mean that resulted from step two of this procedure. During the trimming procedure, means and $S D$ s were calculated using all RTs from all participants, separately for each combination of ability to switch (switcher or perseverator), block (shape, color, or size), subblock, and correctness (correct or incorrect responses). This trimming procedure resulted in the replacement of $6.0 \%$ of trials with a new RT. Trimming successfully removed the influence of extreme RTs and produced equivalent results to those obtained using median RTs.

5. Two participants were excluded from the ANCOVA analysis due to missing RT data.

\section{APPENDIX}

\section{Introduction}

\section{Study Design}

"What happens when you press the pictures? Does the box change color?"

(Experimenter notes that boxes turn green when tapped)

\section{Block 1}

(Thirty-three trials presented, in the following order)

Three target identification trials: "Can you press the cat [bird, fish]?"

Three task introduction trials: "Now we're going to play the shape game. In the shape game, when you see a cat, press the cat. Can you press the cat?" [bird, fish]

Three nonconflict questions: "In the shape game, show me what you press when you see a cat [bird, fish]"

Three visual nonconflict trials: "Okay! Which do you press for this one?"

Six conflict trials: "Okay! Which do you press for this one?"

Three identity trials: "Okay! Which do you press for this one?"

Six conflict trials: "Okay! Which do you press for this one?"

"Good job! Just keep doing what you were doing."

Six novel trials: "Okay! Which do you press for this one?"

\section{Block 2}

(Thirty-three trials presented, in the following order)

Three target identification trials: "Can you press the red one [yellow one, blue one]?"

Three task introduction trials: "Now we're going to play the color game. In the color game, when you see a red one, press the red one. Can you press the red one?" [yellow one, blue one]

Three nonconflict questions: "In the color game, show me what you press when you see a red one [yellow one, blue one]"

Three visual nonconflict trials: "Okay! Which do you press for this one?"

Six conflict trials: "Okay! Which do you press for this one?"

Three identity trials: "Okay! Which do you press for this one?"

Six conflict trials: "Okay! Which do you press for this one?"

"Good job! Just keep doing what you were doing."

Six novel trials: "Okay! Which do you press for this one?"

\section{Block 3}

(Thirty-three trials presented, in the following order)

Three target identification trials: "Can you press the small one [big one, medium one]?"

Three task introduction trials: "Now we're going to play the size game. In the size game, when you see a small one, press the small one. Can you press the small one?" [big one, medium one]

Three nonconflict questions: "In the size game, show me what you press when you see a small one [big one, medium one]"

Three visual nonconflict trials: "Okay! Which do you press for this one?"

Six conflict trials: "Okay! Which do you press for this one?"

Three identity trials: "Okay! Which do you press for this one?"

Six conflict trials: "Okay! Which do you press for this one?"

"Good job! Just keep doing what you were doing."

Six novel trials: "Okay! Which do you press for this one?" 
APPENDIX (Continued)

Stimulus Examples
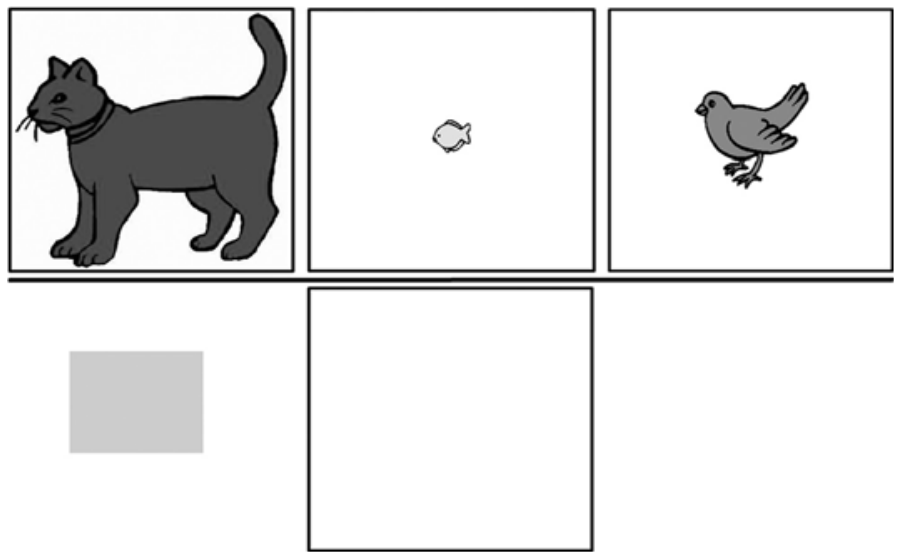

Figure A1. Introduction (before Block 1); target identification trials; nonconflict questions. Dark, medium, and light gray correspond to blue, red, and yellow, respectively.
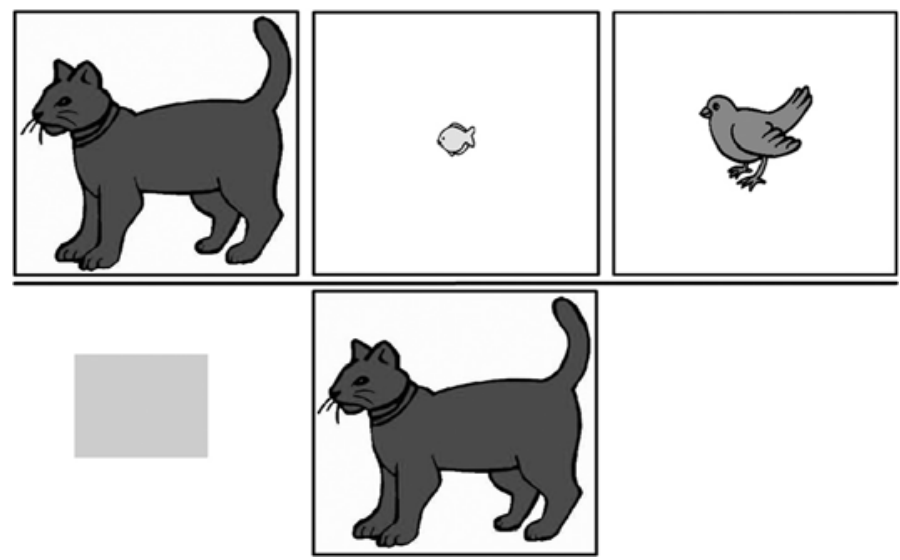

Figure A2. Task introduction trials (e.g., large blue cat presented when video began); identity trials (e.g., large blue cat presented when video ended). Dark, medium, and light gray correspond to blue, red, and yellow, respectively.
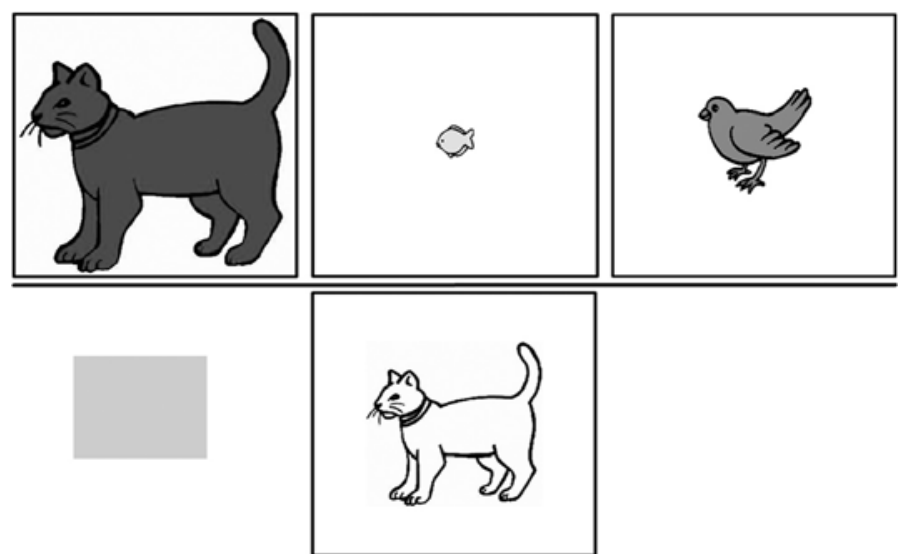

Figure A3. Visual nonconflict trials (e.g., medium-large white cat presented when video ended). Dark gray, medium gray, light gray, and white correspond to blue, red, yellow, and white, respectively. 
APPENDIX (Continued)

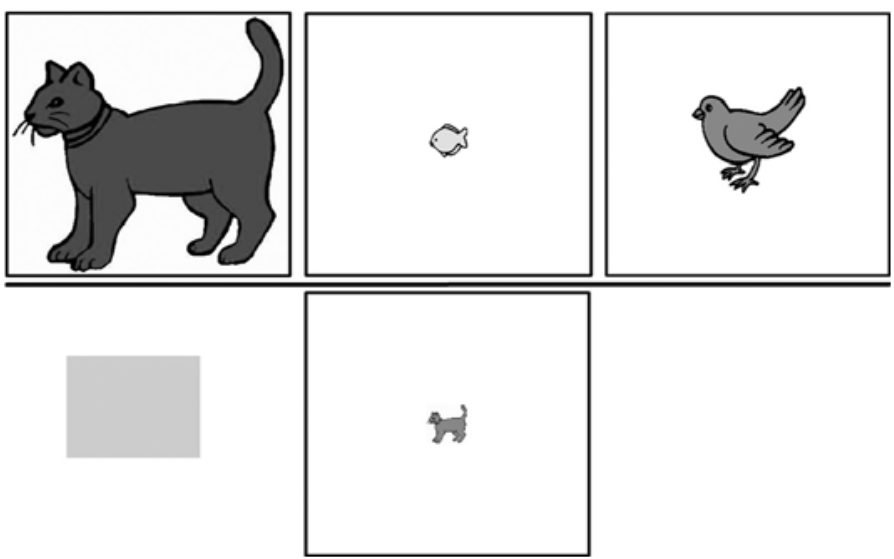

Figure A4. Conflict trials (e.g., small red cat presented when video ended). Dark, medium, and light gray correspond to blue, red, and yellow, respectively.

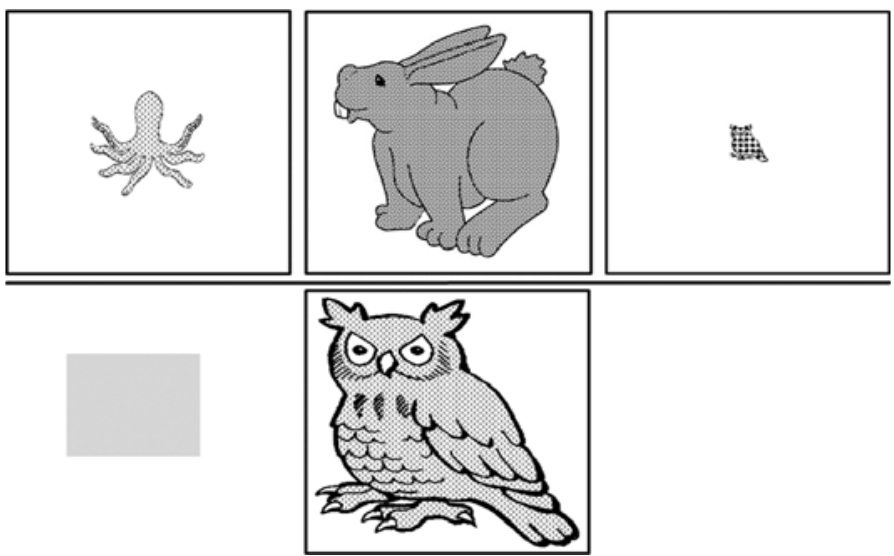

Figure A5. Novel conflict trials (stimulus presented when video ended). Dashes, diamonds, and dots correspond to purple, green, and orange, respectively.

(Manuscript received March 26, 2006;

revision accepted for publication May 4, 2007.) 